\title{
A SEARCH FOR ACOUSTIC AMPLITUDE DEFICIT AT THE ANTIPODES OF SUNSPOTS
}

\author{
MICHAL L. PERI and KEN G. LIBBRECHT \\ Big Bear Solar Observatory, California Institute of Technology, Pasadena, CA 91125, U.S.A.
}

(Received 1 August, 1990; in revised form 4 October, 1990)

\begin{abstract}
We present a search for the acoustic oscillation deficit which may exist at the antipodes of sunspots. Dopplergrams from Big Bear Solar Observatory 1988 helioseismology data were selected for five days on which large sunspots were known to be on the unseen hemisphere of the Sun. Acoustic oscillation amplitudes in the antipodal regions of these sunspots were compared with amplitudes in surrounding quiet-Sun regions. We did not detect a statistically significant acoustic amplitude deficit in our data. Our results indicate that the amplitude deficit at the sunspot antipodal points is limited to no more than $3 \%$ of the acoustic amplitude in the region, for solar oscillation modes of spherical harmonic degree $l \lesssim 200$. We conclude that no strong acoustic deficit exists at the antipodes of sunspots. A more sensitive search, requiring more elaborate observations than we have performed, would be desirable in order to determine if a weak acoustic amplitude deficit exists at some level at the antipodes of sunspots, perhaps at higher spatial frequencies of oscillation. The noise level in any signals detected by such observations would probably limit their usefulness as seismic probes. However, information on the lifetimes of solar oscillation modes can be deduced even if no acoustic amplitude deficit is detected.
\end{abstract}

\section{Introduction}

It is known that sunspots absorb acoustic energy. Studies by Braun, Duvall, and LaBonte $(1987,1988)$ revealed that sunspots act as selective filters of acoustic waves, absorbing $40 \%-50 \%$ of the energy incident into the sunspot in the form of $p$-mode oscillations with spherical harmonic degree $l \gtrsim 200$. Direct measurements of the amplitudes of solar oscillations in an active region by Tarbell et al. (1988) show a suppression of power in $f$ - and $p$-modes which closely follows the magnetic contours of the region. Lindsey and Braun (1990) have proposed that the amplitude deficit generated by an active region could be focused by the spherical symmetry of the Sun as a wavepropagating medium, such that an acoustic amplitude deficit would result at the antipode of a sunspot. They have speculated that the deficit at the antipode could be analyzed to deduce an absorption map of sunspots on the unseen side of the Sun, in analogy to seismic imaging using waves generated in terrestrial earthquakes. Roddier (1975) has suggested that acoustic signals could be used for detecting sub-surface acoustic sources in the Sun through the technique of acoustic holography.

The key to formation of an acoustic signal at the antipode of a sunspot is that oscillation modes having sufficient spatial frequency to interact with sunspots must be coherent for at least the amount of time required to propagate to the antipode. As pointed out by Lindsey and Braun (1989), the magnitude of the acoustic deficit at the antipode, and the contrast and profile of the image which can be derived, depends on the lifetimes of the oscillation modes. Even if an acoustic deficit is not found at the antipodal points it should be possible to put limits on the mode lifetimes. Thus the search 
for an acoustic deficit at the antipodes of sunspots will be useful whether or not it is found.

We have conducted such a search using helioseismology data acquired during the summer of 1988 at Big Bear Solar Observatory (BBSO). Five days were selected from the helioseismology data set for which a large sunspot group was known to be located on the unseen hemisphere of the Sun and the locations of the antipodes of these sunspots were unobscured by active regions. Dopplergrams taken on these five days were averaged to produce daily maps of the root-mean-square velocity amplitude on the face of the Sun. We looked for an acoustic deficit by comparing the velocity amplitude in the vicinity of the antipodes of the sunspots to that in surrounding quiet-Sun regions.

\section{Data Acquisition and Prelimimary Reduction}

The data used in this analysis were selected from data acquired on 129 days during April-September 1988 by the dedicated helioseismology telescope at BBSO. Observations were made with a tunable Zeiss $0.25 \AA$ filter centered on the $6439 \AA$ solar calcium line. A KDP electro-optical crystal was used to shift the bandpass by $\pm \frac{1}{8} \AA$. Full disk solar images in the red and blue wings of the line were acquired at video rates with a Cohu CCD camera and digitized to $480 \times 512$ pixels with eight bits. Red/blue image pairs were subtracted, then 752 such pairs were averaged to produce full-disk Dopplergrams with pixel levels separated by approximately $10 \mathrm{~m} \mathrm{~s}^{-1}$. These Doppler images were collapsed down to $192 \times 240$ pixels, one byte deep, before being stored to tape. This process was repeated once per minute. The telescope and data acquisition system are described in detail by Libbrecht and Zirin (1986) and Libbrecht (1988).

Preliminary data reduction began with rescaling the data to $128 \times 128$ pixels and centering each image. The images were then derotated and scaled to a uniform size. Bad points were removed from the data set by fitting small sections of images $(32 \times 32$ pixels) to a mean velocity plus solar rotation and manually examining the time series of fit coefficients. Cloud gaps in the data were smoothed over by interpolation between adjacent good images. Then an average Dopplergram computed from the running mean of the 20 nearest-neighbor images was subtracted from each adjusted final image. This was done to remove slow trends and the effects of solar rotation from the data (Libbrecht and Zirin, 1986). Finally, a quadratic fit for the limb darkening at $\lambda=6439 \AA$ was calculated from

$$
\frac{I(\mu)}{I(0)}=0.3701+0.9149 \mu-0.2859 \mu^{2}
$$

where $\mu=\cos (\theta)$. The coefficients have been interpolated from those tabulated by Pierce and Slaughter (1977). The velocity amplitude at each pixel was divided by $\mu I(\mu) / I(0)$ to correct for limb darkening and projection.

The corrected Doppler images thus produced measure the velocity amplitude of the solar oscillations at each point on the Sun at 1-min intervals. For each day of observations a pointwise root-mean-square average was computed from all the Dopplergrams 
for that day, usually more than 600 frames, to produce a $128 \times 128$ pixel map of the daily average acoustic oscillation amplitude on the face of the Sun. The mean value in a central region of radius 200 arc sec was then calculated for each daily image. These central mean values were tabulated and images with exceptionally high central means were rejected. Exceptionally high central mean values indicated an irregularity of the data occurring in unusually grainy daily acoustic images. This was found to correlate with the number of individual Dopplergrams in the daily data set, in that days with less data (due to cloud gaps, etc.) produced higher central mean values. The remaining images were scaled so that the acoustic amplitude in the central region of radius 200 arc sec had a mean value of 100.00 in each daily acoustic map.

The daily acoustic amplitude images were then displayed for visual inspection. The images were compared with the daily sunspot maps produced at BBSO. It was found that large active regions with one or more sunspots consisting of both umbra and penumbra (identified from the sunspot maps and corresponding broadband images taken in $\mathrm{H} \alpha$ ) were readily identified with features in the average daily acoustic maps. The coordinates of these features were checked against the tabulated daily coordinates of BBSO active regions.

\section{Acoustic Amplitude Measurements}

In order to search for an acoustic signal at the antipode of a sunspot, it was necessary to identify days in which a sizeable sunspot group occurred in a known location on the back of the Sun. To minimize limb-darkening and projection effects it was desirable that the antipode of the sunspot occur near the solar meridian. Moreover, it was necessary that there be no active region in the vicimity of the antipode on the front of the Sun. Proximity of an active region to the antipode would complicate the observations because oscillation amplitudes are diminished in regions with high magnetic fields (Tarbell et al., 1988). Also, photospheric motions in and around sunspots, combined with seeing effects, could confuse the observations.

To select such days, the data set of acoustic images was separated into subsets consisting of daily acoustic maps at intervals of 14 days, or roughly one-half the solar rotation period in the sunspot latitudes. Acoustic images were then manually selected from these subsets by the following criteria: (1) both the preceding and the following images (one-half solar rotation period earlier and later, respectively) must show an active region near the solar meridian with at least one large sunspot possessing a welldeveloped umbra and penumbra; (2) there should be enough similarity of appearance in the preceding and following images to convincingly be two visitations of the same active region; (3) the acoustic image must have no active region in the vicinity of the antipode.

Five days were selected by these criteria from the 1988 helioseismology data set for further analysis. On each of these days a large sunspot group was known to be located on the back of the Sun. The position of the active region on the back of the Sun was calculated by interpolation from the observed positions of the active region on the front 


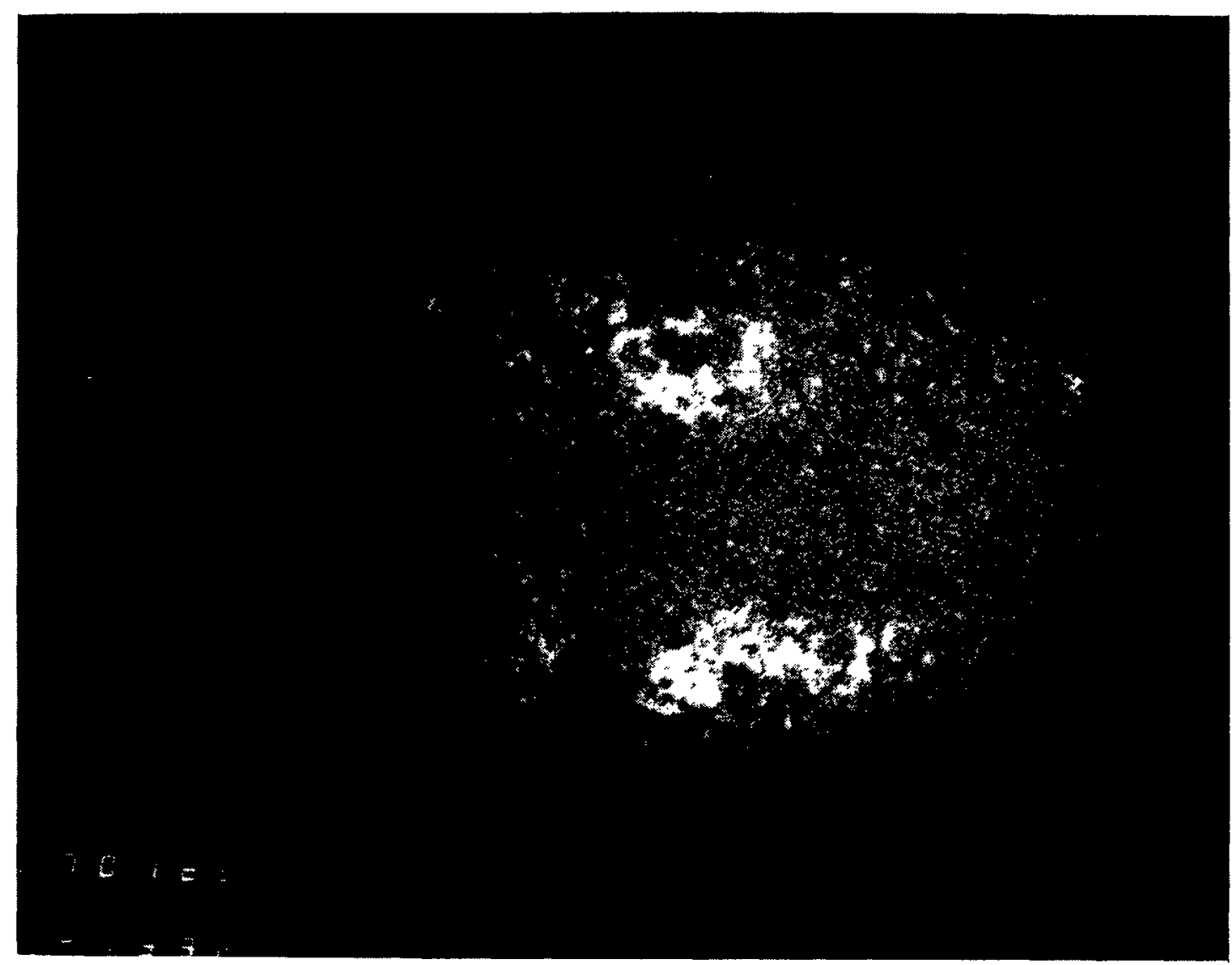

Fig. 1. H $\alpha$ image of the Sun taken on July 1, 1988. The large active region with prominent sunspot appearing near the meridian in the south is BBSO No. 1167. The same sunspot group crossed the meridian again on July 29,1988 . We were thus able to interplate the position of the sunspot while it was on the back of the Sun.

of the Sun in the preceding and following images. This determined the position of the antipode in the acoustic image of the selected day. We estimate the error in the position of the antipode to be less than two pixels ( $30 \mathrm{arc} \mathrm{sec})$. Figure 1 shows one of the sunspot groups studied, which crossed the meridian of the Sun on July 1, 1988. Figure 2 shows the antipodal region of that sunspot as it crossed the meridian (in the northern hemisphere of the Sun) on July 15, 1988. Both images were taken in $\mathbf{H} \alpha$.

To determine whether an acoustic deficit is produced at the antipode of a sunspot one must sample the velocity amplitude in the vicinity of the antipode and compare it with the velocity amplitude in regions of the Sun which are removed from sunspots and their antipodes. Ideally, one would compare the region containing the antipode with symmetrically-spaced regions which do not contain sunspots or their antipodes. Unfortunately, asymmetries in the Zeiss filter made it impractical to compare regions on opposite sides of the solar disk. We therefore chose to compare concentric regions centered on the antipode to search for a signal. It was necessary to compromise between choosing regions sufficiently large to encompass the acoustic deficit and containing enough points to produce small standard deviations, yet not so large that a small signal would be averaged out. Lindsey and Braun (1989) claim that in a spherically symmetric 


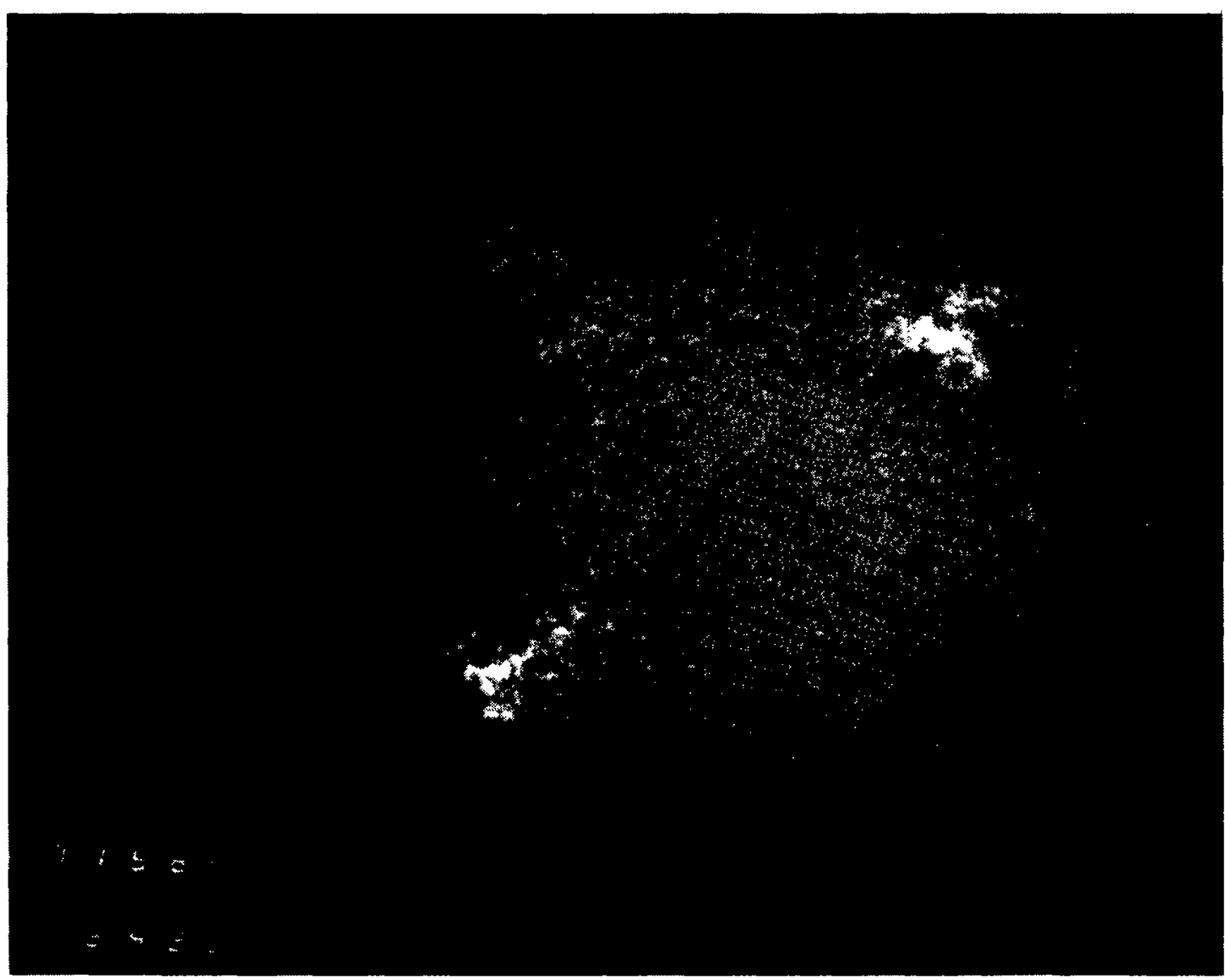

Fig. 2. H $\alpha$ image of the Sun taken on July 15,1988 . On this day the antipode of the sunspot shown in Figure 1 crossed the meridian in the northern hemisphere. The location of the antipode on the front of the Sun was devoid of solar activity. The sunspot group appearing in the southeast is BBSO No. 1175. Its antipode had crossed the solar meridian on July 4, 1988 and was also included in our analysis.

Sun the acoustic waves should be strongly focused at the antipode of a point absorber. This was interpreted to mean that, in the perfect case, the linear extent of the signal at the antipode would be comparable to the wavelength of the highest $l$ mode imaged, taken to be two resolution elements. For images with $128 \times 128$ pixels, $l_{\text {max }}=2 \pi(64$ pix $) /(2$ pix $) \approx 200$ which corresponds to a wavelength of $\lambda \approx 30$ arc sec.

For our analysis we chose to sample three concentric regions. The first region, a circle of radius $60 \mathrm{arc} \mathrm{sec}$, is a factor of four larger than the minimum calculated for the perfect case above. This was chosen to allow for defocusing by deviations in the Sun from a perfect spherically-symmetric wave propagating medium, and also allowed for blurring due to solar rotation introduced by averaging images over a full day's observing time. At sunspot latitudes this blurring corresponds to roughly $80 \mathrm{arcsec}$ over 10 hours. Moreover, a diameter of $120 \mathrm{arc} \mathrm{sec}$ is commensurate with the diameters of the large sunspot groups used. Finally, this region contained a sufficient number of pixels to provide a statistically meaningful measure.

The second and third regions consisted of annuli centered on the antipodes with outer radii of $120 \mathrm{arcsec}$ and $180 \mathrm{arcsec}$, respectively. These provided a measure of the acoustic amplitude in areas of quiet Sun away from the antipode. The second ring was 
used to look for any diffuse acoustic signal not contained in the central circle. The third ring was taken so large that any remaining acoustic signal would be overwhelmed by background. Figure 3 shows a Dopplergram at the antipode of the sunspot group shown in Figure 1 overlaid by a diagram of the three sampling regions. A comparison can be made with same region imaged in $\mathrm{H} \alpha$ in Figure 2.

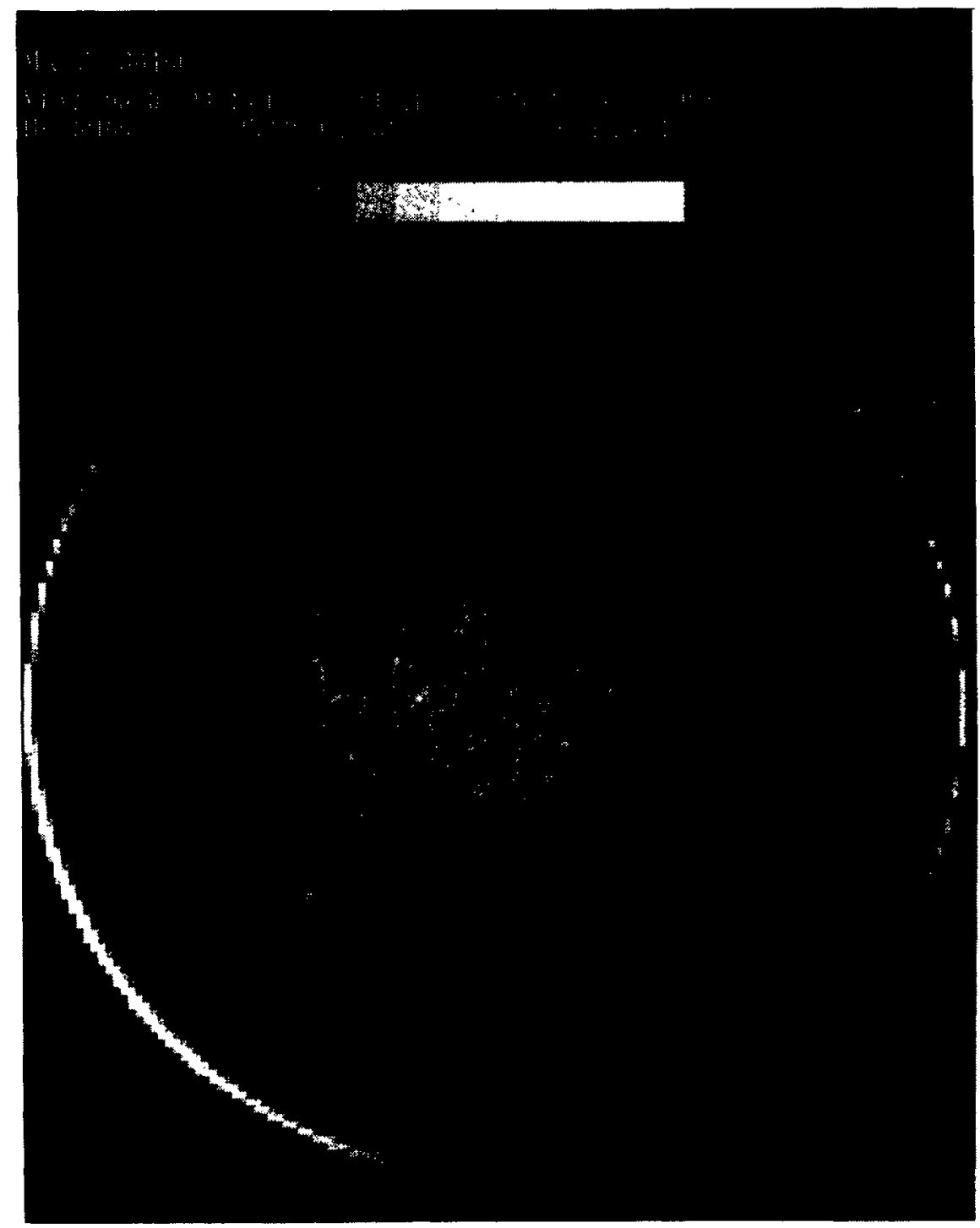

Fig. 3. Average daily Dopplergram of the Sun (derotated and scaled) taken for July 15, 1988, overlaid by a diagram of the three sampling regions. This image can be compared with the $\mathrm{H} \alpha$ image shown in Figure 2. Upon inspection, one can see that no 'shadows' are visible in the location of the antipode. However, the sunspot group in the southeast clearly generates an acoustic signal.

\section{Results}

The mean and standard deviation of the acoustic amplitude in the three regions was calculated for each of the five chosen days. The results are presented in Table I. Note that data were scaled such that the acoustic amplitude in a central region of radius 
200 arc sec had a mean value of 100.00 in each daily acoustic map, and that limb darkening and projection effects have been removed from the data.

Inspection of the statistics in Table I reveals that a large acoustic signal at the antipode does not occur. The difference in acoustic amplitude between the inner and outer rings

TABLE I

Acoustic amplitude measured at the antipodes of sunspots

\begin{tabular}{|c|c|c|c|c|c|c|c|c|}
\hline \multirow[t]{2}{*}{ Date } & \multicolumn{2}{|c|}{ Antipode } & \multirow{2}{*}{$\begin{array}{l}\text { 1st region } \\
\text { (center) }\end{array}$} & \multirow{2}{*}{$\begin{array}{l}\text { 2nd region } \\
\text { (inner ring) }\end{array}$} & \multirow{2}{*}{$\begin{array}{l}\text { 3rd region } \\
\text { (outer ring) }\end{array}$} & \multicolumn{3}{|c|}{ Differences } \\
\hline & $\lambda$ & $\phi$ & & & & $1 \mathrm{st}-2 \mathrm{nd}$ & $2 \mathrm{nd}-3 \mathrm{rd}$ & $1 \mathrm{st}-3 \mathrm{rd}$ \\
\hline 051488 & -23 & 5 & $100.66 \pm 0.75$ & $98.49 \pm 0.44$ & $99.03 \pm 0.43$ & 2.16 & 1.62 & -0.54 \\
\hline 062088 & 26 & 19 & $97.39 \pm 0.96$ & $94.54 \pm 0.50$ & $94.69 \pm 0.50$ & 2.84 & 2.69 & -0.15 \\
\hline 070488 & -18 & 4 & $93.77 \pm 0.73$ & $94.19 \pm 0.45$ & $93.86 \pm 0.43$ & -0.42 & -0.09 & 0.33 \\
\hline 071588 & -20 & 2 & $96.68 \pm 0.69$ & $94.24 \pm 0.42$ & $96.22 \pm 0.37$ & 2.44 & 0.47 & -1.97 \\
\hline 072788 & 22 & 7 & $100.88 \pm 0.65$ & $102.22 \pm 0.44$ & $102.39 \pm 0.38$ & -1.34 & -1.51 & -0.17 \\
\hline
\end{tabular}

is close to $1 \sigma$, hence there is no evidence for a diffuse signal extending beyond a radius of $60 \mathrm{arcsec}$. A difference at the $3 \%$ level does exist between the central region containing the antipode and the inner ring. However this signal occurs in the sample as both a deficit and an excess of velocity amplitude, and is not statistically significant. It should also be noted that no 'shadows' are visible in antipodal regions on the daily acoustic maps (see Figure 3). Hence, if an acoustic deficit occurs at the antipode it must be diffuse over an area of radius greater than $200 \mathrm{arc} \mathrm{sec}$. Such a signal would necessarily be faint, and could not readily be used for acoustic imaging of sunspots on the back of the Sun.

\section{Discussion and Conclusions}

In this section we discuss possible explanations for our lack of observation of a strongly focused acoustic deficit at the sunspot antipodal points. The first explanation is that the acoustic amplitude deficit may be not be created in the range of spatial frequencies sampled by our observations. Braun, Duvall, and Labonte $(1987,1988)$ found that sunspots absorb up to $50 \%$ of the incident acoustic energy in oscillation modes with spherical harmonic degree $l \gtrsim 200$, but that the amount of absorption falls for oscillations of lower spatial frequency. We would be unable to detect absorption in modes with $l \gtrsim 200$, since they are beyond the sensitivity of our observations. However, one should note that the reported observations were performed on sunspots of diameter $\lesssim 25$ arc sec. The sunspots we studied were many times larger, with semi-major axes typically $\gtrsim 50 \mathrm{arc} \mathrm{sec}$. It is reasonable to suppose that such larger sunspots could interact with lower $l$ oscillation modes. Thus it is possible that the larger sunspots we studied could produce significant absorption of oscillation modes sampled by our observations.

An alternate interpretation of the lack of a strong acoustic deficit at the antipodes of 
sunspots is that absorption of $l \lesssim 200$ solar oscillation modes does occur in sunspots, but that no focused acoustic amplitude deficit forms at the antipodal point. This would suggest that the lifetime of the high-frequency $p$-modes which interact with sunspots is not long enough for the waves to propagate to the antipode. If we assume negligible dissipation in the quiet Sun, then a simple calculation allows us to place limits on the lifetimes of solar oscillation modes. The coherence lifetime of an oscillation mode must be less than one half of the circumference of the Sun divided by the horizontal group velocity. The horizontal group velocity is equal to $\partial v / \partial l$ times a scaling factor equal to the solar circumference. Values of $\partial v / \partial l$ have been tabulated by Libbrecht, Woodard, and Kaufman (1990). For $l=200, \partial v / \partial l=5 \mu \mathrm{Hz}$, which implies a lifetime $\lesssim 10^{5} \mathrm{~s}$. This is consistent with estimates of the mode lifetimes derived from the width of observed ridges in an $l-v$ diagram (K. G. Libbrecht, unpublished results).

It should be possible to perform additional observational studies which can discriminate between the two cases discussed above. Observations similar to those described by Braun, Duvall, and LaBonte (1988) could be performed on a wider variety of active regions to determine the dependence of absorption on sunspot geometry and on the spatial frequency of the oscillations. Such a study could advance development of theories of the mechanism for $p$-mode absorption in sunspots, which is poorly understood at this time. It could also yield information on the subsurface structure of solar active regions. In particular, this should determine whether larger sunspots such as those observed in this work absorb solar oscillation modes with lower spatial frequencies.

It would also be desirable to perform additional observations with higher spatial resolution at the antipodes of sunspots. This would allow us to look for an acoustic amplitude deficit in oscillation modes which we know to be absorbed efficiently by sunspots. One could also perform temporal filtering on the data to isolate the 5-min oscillations by suppressing low temporal frequency noise. Other filtering procedures which may enhance the probability of detecting a weak acoustic amplitude deficit at the antipode are discussed by Lindsey and Braun (1990). Since the absorption of such modes by sunspots has been measured, such observations should bear directly on the mode lifetimes and on their attenuation in the quiet Sun.

\section{Acknowledgements}

We are grateful to the observing staff at BBSO for their support in these observations, and to Dr. Martin Woodard for his enlightening comments. The work at BBSO is supported by the National Science Foundation under contracts ATM-8604632 and AST-8657393.

\section{References}

Braun, D. C., Duvall, T., and LaBonte, B. J.: 1987, Astrophys. J. 319, L27.

Braun, D. C., Duvall, T., and LaBonte, B. J.: 1988, Astrophys. J. 335, 1055.

Libbrecht, K. G.: 1988, Astrophys. J. 334, 810.

Libbrecht, K. G. and Zirin, H.: 1986, Astrophys. J. 308, 413. 
Libbrecht, K. G., Woodard, M. F., and Kaufman, J. M.: 1990, Astrophys. J. Suppl. 74, 1129.

Lindsey, C. and Braun, D. C.: 1991, Solar Phys. (submitted).

Pierce, A. K. and Slaughter, C. D.: 1977, Solar Phys. 51, 25.

Roddier, F.: 1975, Compt. Rend. Acad. Sci. 281, B.933.

Tarbell, T., Peri, M., Frank, Z., Shine, R., and Title, A.: 1988, in E. J. Rolfe (ed.), Seismology of the Sun and Sun-Like Stars, ESA Publ. Div., ESTEC, Noordwijk, The Netherlands, p. 315. 